\title{
TEATRO 2 - UM TELETEATRO DE EXPERIMENTAÇÃO, DIFUSÃO E RESISTÊNCIA
}

\author{
THEATER 2 - A TELETEATRO TESTING, \\ DISSEMINATION AND RESISTANCE
}

\begin{abstract}
ANTONIO DE ANDRADE, Cicilia K. Peruzzo e SANDRA Reimão

Antonio de Andrade possui mestrado em Comunicação Social pela Universidade Metodista de São Paulo (1982). Atualmente é professor Adjunto da Universidade Metodista de São Paulo e Coordena o Curso de Radialismo na Faculdade de Comunicação da UMESP.

Cićlia K. Peruzzo possui graduação em Comunicação Social pela Faculdade de Comunicação Social Anhembi, mestrado em Comunicação Social pela Universidade Metodista de São Paulo e doutorado em Ciências da Comunicação pela Universidade de São Paulo. Atualmente é professora do Programa de Pós-Graduação em Comunicação da Universidade Metodista de São Paulo.

Sandra Reimão é professora do Programa de Pós Graduação em Comunicação da Universidade Metodista de São Paulo e bolsista de Produtividade em Pesquisa do CNPq. Diretora adjunta da Cátedra Unesco de Comunicação para o Desenvolvimento Regional / Metodista. Graduada em Filosofia pela Universidade de São Paulo - USP. Mestre e Doutora em Comunicação pela Pontifícia Universidade Católica de São Paulo - PUC-SP.
\end{abstract}

\section{RESUMO}

O artigo retoma uma época importante da história da televisão brasileira, o início das transmissões televisivas em 1950, e em especial, a transmissão dos teleteatros, pois estes programas foram os mais prestigiados pela televisão daquela época, tornando-se mesmo a própria definição da televisão na década de 1950. Através de pesquisa bibliográfica, foi identificado que o declínio do teleteatro aconteceu a partir de 1962, com a chegada do videotape e, com ele, o surgimento das telenovelas diárias que cada vez mais ganharam espaço entre os telespectadores. Sob a perspectiva da importância dos teleteatros para a televisão brasileira, surge em 1974 o projeto Teatro 2 na Televisão Cultura, sob a criação de Nydia Lycia. Esse projeto se tornou um dos mais bem sucedidos na busca da reintegração dos teleteatros na programação televisiva, trazendo adaptações de textos literários importantes para a TV. Contribuindo para o aumento e incentivo do interesse do público para a cultura teatral e a literária, além de proporcionar abertura da televisão para vários outros segmentos culturais e estéticos.

Palavras-chave: Teleteatro, Teatro 2, Televisão Brasileira 


\section{ABStRACT}

The article takes an important time in the history of Brazilian television, the start of television broadcasting in 1950, and in particular the transmission of teleteatros, since they were the most prestigious television at that time, even becoming the very definition television in the 50's. Through literature review, it was reported that the decline of teleteatro happened since 1962, with the advent of videotape and with it the emergence of daily telenovelas that increasingly became more popular with viewers. From the perspective of the importance of teleteatros for Brazilian television, appeared in 1974 the project Theater 2 TV Cultura, in the creation of Nydia Lycia. This project became one of the most successful in the pursuit of the reintegration of teleteatros in television programming, bringing adaptations of literary texts relevant to the TV. Contributing to the increase and encouragement of public interest for the theatrical and literary culture, and provide open television to other segments of various cultural and aesthetic.

Keywords: Teleteatro, Theater 2, TV Brasileira

\section{Teleteatro - um panorama}

O início das transmissões televisivas regulares no Brasil se deu em 18 de setembro de 1950, com a inauguração da emissora TV Tupi em São Paulo. Poucos meses depois, em 29 de novembro, temse a exibição do primeiro teleteatro - A vida por um fio, na realidade a tradução e adaptação de um filme da Paramount de 1948, dirigido por Anatole Litvak, Sorry, wrong number. Esse filme, por sua vez, era baseado em um programa de rádio da CBS (PORTO E SILVA, 1981).

Durante toda primeira década de sua existência, a televisão brasileira, assim como em outros países (BRANDÃO FARIA, 2005), teve no teleteatro seu ponto máximo de prestígio cultural: "Criado em 1950, o teleteatro se firma nos primeiros anos de sua existência para se con- sagrar como o programa de maior prestígio produzido pela televisão brasileira" (ORTIZ, 1991, p. 43).

Dentre os programas de teleteatro do início da televisão brasileira os mais marcantes foram: TV de Vanguarda, na TV Tupi, e o Grande Teatro Tupi, também na mesma emissora.

O programa TV de Vanguarda permaneceu no ar entre 1952 e 1967 (BRANDÃO FARIA, 2005) e como explica Inimá Simões, inicialmente denominava-se Teatro de Vanguarda e concedeu "[...] prestígio à emissora e se tornará para muitos a própria definição da televisão dos anos 50, assim como a novela será sem dúvida a sua expressão mais completa nas décadas seguintes (SIMÕES, 1986, p. 29)." Em seguida, Inimá Simões detalha que em geral a postura mais freqüente diante da TV de Vanguarda é considerar 'o programa como 
algo irretocável e vê com nostalgia o alto nível conseguido nas encenações que levavam ao ar autores como Brecht, Goethe, Dostoievsky, Pirandello, Steinbeck, Maughan, Shakespeare, Lorca" (SIMÕES, 1986, p. 29).

O Grande Teatro Tupi foi transmitido pela TV Tupi de São Paulo entre novembro de 1951 e 1965 (PORTO E SILVA, 1981) e pela TV Tupi do Rio de Janeiro entre 1956 e $1965^{1}$.

No livro "Almanaque da TV", Ricardo Xavier - Rixa descreve o início do Grande Teatro Tupi em São Paulo assim:

Em 21/5/51, a Tupi paulista anunciou uma programação de teleteatros, estreando com a montagem de "Professor de Astúcia”, espetáculo que estava em cartaz na cidade. [...] $O$ programa vingou [alguns meses mais tarde] com o nome de "Grande Teatro das SegundasFeiras", transmitido no dia de folga das companhias teatrais. Patrocinado pela construtora imobiliária Monções, a atração foi rebatizada de 'Grande Teatro Monções', título que mudava a cada novo anunciante [...] o programa entrou para a história como o "Grande Teatro Tupi”. (RIXA, 2000, p. 111)

O Grande Teatro Tupi no Rio de Janeiro é filho direto e explícito do Grande Teatro Tupi de São Paulo e 1 Brandão, Cristina. Obra citada pg. 14. Detalhando: "O Grande Teatro Tupi foi exibido todas as segundas- feiras de 1956 a 1962 na TV Tupi, de 63 a 64, na TV Rio e alguns meses na estreante TV Globo, em 1965". só surgiu seis anos depois deste:

Para falarmos do Grande Teatro Tupi, no Rio de Janeiro, temos que retomar suas origens, na PRF-3 TV Tupi de São Paulo, aonde, em 1951, algumas companhias e grupos teatrais, tais como a Sociedade Paulista de Teatro e o Teatro de Arte, vinham se apresentando de forma esporádica na emissora. A SPT, fundada e dirigida pela atriz Madalena Nicol, foi uma sociedade patrocinada pelo Governo de São Paulo e reunia nomes como Ruggero Jaccobi, Jaime Barcelos, Armando Couto, Carla Civelli, Sérgio Britto, Xandó Batista e Silvia Orthof. Em novembro daquele ano, a Tupi começou a apresentar, regularmente, um "grande teatro" às segundas-feiras. A idéia não era original, pois mais uma vez a televisão vai buscar no rádio elementos para se basear ao estruturar sua programação. $O$ próprio nome não era novidade, pois o Grande Teatro Tupi tinha sido um radioteatro famoso da Rádio Tupi assim como o Cinema em Casa na Rádio Difusora, criados por Otóvio Gabus Mendes e que rivalizavam com o tradicional Teatro Manuel Durães (na Rádio Record), produzido por Manuel Durães (BRANDÃO FARIA, 2002).

O Teatro Tupi do Rio de Janeiro transmitiu, ao vivo, mais de 400 encenações baseadas em peças, romances e contos, de autores brasileiros e estrangeiros, como Flaubert, Goethe, Dostoievsky, Tennessee Williams, Machado de Assis e José de Alencar. No elenco, atores como: Fernanda Montenegro, Nathalia Timberg, Ítalo Rossi, Sergio Britto, Leonardo Villar, Francisco Cuoco, Aldo de Maio, Berta Zemel, Milton Moraes e outros. Como diretores: Fernando Torres, Flávio Rangel e Sergio Brito. Manoel Carlos, que adaptou quase 100 textos para o Grande Teatro Tupi explica: "Participar de nosso grupo era o mesmo que participar, por exemplo, do 
elenco do Teatro Brasileiro de Comédia, o TBC, a companhia teatral mais importante e respeitável da época". ${ }^{1}$

\section{Teleteatro e telenovela}

Com o advento das gravações em videotape, a partir de 1962, surgem as telenovelas diárias, a ficção televisiva seriada com capítulos diários, e essas telenovelas vão se consolidando na preferência do telespectador. "Na verdade, a telenovela torna-se o produto através do qual os canais concorrem entre si; todas as emissoras tentam uma incursão pelo gênero" (RAMOS; BORELLI, 1991, p. 63). Com a consolidação da telenovela, os teleteatros vão sendo desativados. Em 1963 a TV Excelsior encerra as transmissões dos programas Teatro 9 e Teatro 63; em 1964 a TV Tupi encerra o Grande teatro Tupi e em 1967 foi realizada a última transmissão do TV de Vanguarda, "encerrando definitivamente um ciclo de teleteatro, e iniciando a era da hegemonia da telenovela" (RAMOS; BORELLI, 1991, p. 63).

Renata Pallottini (1998, p. 25) define os programas unitários, entre os quais, o teleteatro, como "uma ficção para TV, levada ao ar de uma só vez com duração de aproximadamente uma hora, programa que se basta em si mesmo, que conta uma história com começo, meio e fim, que esgota sua proposição na unidade e nela se encerra".

Cristina Brandão Faria utiliza termos análogos:

Defino por teleteatro, uma representação dramática transmitida pela televisão, reunindo em uma única apresentação, uma história com princípio, meio e fim. Um espetáculo de teleteatro pode variar entre a exibição de um texto teatral adaptado para a televisão (geralmente, a maioria), um conto, uma obra literária ou também um filme. (BRANDÃO FARIA, 2005, p. 53)

$\mathrm{O}$ advento da telenovela diária (1963), o cerceamento da censura durante a Ditadura Militar (19641985), o sucesso do filme estrangeiro, o constante crescimento dos custos de produção, estão entre os fatores que podem servir de chave explicativa para o declínio do teleteatro. Muitos acreditam que o teleteatro não acabou e sim que foi incorporado pela telenovela:

Alijado da programação pela telenovela e pelo filme estrangeiro, cerceado pela censu$\mathrm{ra}$, o teleteatro não desapareceu de vez do vídeo. Algumas tentativas esparsas e sem continuidade foram ainda feitas após o termino dos grandes programas do gênero. Para alguns, o teleteatro, na verdade, não teria acabado; ele teria sido absorvido pela novela diária, que para si desviou os recursos de produção das emissoras, valendo-se inclusive dos mesmos produtores, autores, artistas $e$ técnicos (PORTO E SILVA, 1981, p. 89).

\section{O programa Teatro 2}

O programa Teatro 2, projetado e criado por Nydia Lycia (PORTO E SILVA, 1981, p. 89) em 1974 na Televisão Cultura emissora da Fundação Padre Anchieta do Esta
2 Manoel Carlos. $O$ Grande teatro. Livreto de apresentação da exposição O Grande Teatro Tupi. Caixa Econômica Fedeal. Exposição de 31 de maio de 2005 a 31 de julho de 2005. Curadoria: Anna Vacchiano. 
do de São Paulo, foi uma das mais bem sucedidas iniciativas no sentido de retomar os teleteatros no interior da programação televisiva nacional.

O Teatro 2 teve início em junho de 1974 com a gravação de maneira experimental da peça Chapetuba Futebol Clube de Oduvaldo Vianna Filho, dirigida por Antunes Filho. A peça foi exibida em 23 de novembro de 1974. Entretanto, não há documentação a respeito dessa exibição, e nem a gravação foi preservada. Assim, os registros históricos acerca do Teatro 2 começam, de fato, com a exibição do teleteatro O Enfermeiro baseado em conto de Machado de Assis exibido em 10 de junho de 1974 (FERRARA, 1981, p. 88).

A idéia inicial de se realizarem adaptações de peças de textos literários para a TV estava fundamentada em uma orientação de cunho cultural, que de resto já era a orientação geral da TV Educativa. Pensava-se, assim, em levar ao conhecimento do público, obras literárias consideradas culturalmente importantes graças ao seu valor artístico (FERRARA, 1981, p. 88).

O objetivo no que diz respeito à formação cultural do telespectador era despertar o gosto pela leitura, no caso das adaptações das obras literárias, despertando também o gosto pelo teatro, incentivando o público a ver teatro.

O produtor Palma Travassos (1981, p. 93) esclarece também que a escolha dos textos era basicamente do arbítrio do diretor, e que "não existe nenhuma tentativa de padronização e homogeneização dos textos". O Teatro 2, segundo
Flavio Porto e Silva (1981, p. 89), "na tradição dos bons teleteatros [...] esse programa serviu de laboratório para alguns diretores, sobretudo Antunes Filho e Adhemar Guerra [...]".

Antunes Filho relembra esse período como um momento de experimentações e, no seu caso, de ênfase no teatro nacional:

Naquela época nós fazíamos teleteatro na TV Cultura. (...) Cada um fazia uma peça por mês, pra ganhar um dinheirinho por mês. Cada semana era um que fazia um grande espetáculo. E eu iniciei com os brasileiros: Rubem Fonseca, Jorge Andrade, Roberto Gomes... Eu fazia o que podia do Brasil. Levantar o Brasil. E Nelson Rodrigues, evidentemente... Vianinha, tudo que era brasileiro eu fazia. Mensalmente eu fazia um brasileiro. Me interessava levar a dramaturgia do Brasil, na época e nós tínhamos uma liberdade para fazer...Era incrivel! Hoje em dia não daria mais pra fazer esse Vestido de Noiva, porque a coisa agora é muito comercial e na época, pra conseguir até o Vestido de Noiva eu estourei o tempo. Eu teria uma semana e eu fiz em quinze dias, estourando o tempo. Foi uma calamidade, mas era permitido. Essas coisas, esses deslizes eram permitidos. Coisas que hoje em dia não é possivel. A produção não deixa mais...Tantoéqueme gozavam... Quando me chamavam na TV Cultura, na sala de produção, o produtor me dizia: "Não me venha com idéias!" É incrivel! Hoje, a gente paga pras pessoas terem idéias e lá...Nós quatro: O Abujamra, o Cassiano e o Ademar, nós brincávamos 
muito com a expressão...Com o que seria a TV... Nós queríamos brincar, estudar o que era TV. E foi nesse rol de coisas, nesse momento, que eu consegui fazer o Vestido de Noiva, estourando o tempo, estourando a verba de produção... Nós contávamos com uma pessoa nos apoiando muito que era a Nídia Lícia, que tomava conta do departamento de teleteatro. Então, ela nos dava muita força e foi possível realizar, fazer um pouquinho mais... Hoje não é possivel! Estourando mais o tempo e a ver- ba, foi possivel naquele tempo. Então foi uma TV que eu chamo de TV amadora. Hoje em dia é muito profissional. (ANTUNES FILHO apud TRAVASSOS, 1981, p. 83)

O programa Teatro 2 foi produzido e exibido pela Televisão Cultura de São Paulo - Canal 2, de 1974 e 1979, com incursões posteriores em 1985, e com reprises esporádicas durante toda a década de 1990 e 2000 . Foram 122 programas.

\section{TEATRo 2 DATA/Títulos/DIRETORES - TOTAL 122}

\begin{tabular}{|c|c|c|}
\hline Data & $\begin{array}{c}\text { TíTULO, AUTOR (LOCAL E DATA DE NASCIMEN- } \\
\text { TO E MORTE) }\end{array}$ & DiRETOR \\
\hline 1974 & $\begin{array}{c}\text { “O Homem Que Galopava”, DE Luís JARDim } \\
\text { (PE, } 1901 \text { - RJ, 1987) }\end{array}$ & FERnando Faro \\
\hline 1974 & $\begin{array}{l}\text { "No Vale do Diabo", DE J.W. SYNGe (IR- } \\
\text { LANDA, 1871-1909) }\end{array}$ & ANTUNES FiLHO \\
\hline 1974 & $\begin{array}{l}\text { "NoITE", DE ÉRICO VERÍSSIMO } \\
\text { (RS, } 1905 \text {-1975) }\end{array}$ & Benjamin Cattan \\
\hline 1974 & $\begin{array}{l}\text { “As CADEIRAS", DE EUGENE IONESCO } \\
\text { (RoMÊNIA, } 1912 \text { - PARIS, 1994) }\end{array}$ & Antonio AbuJAmRA \\
\hline 1974 & $\begin{array}{c}\text { “O Anúncio Feito a Maria”, de } \\
\text { Paul Claudel (França, } 1868-1955 \text { ) }\end{array}$ & Antonio AbuJAmRA \\
\hline 1974 & $\begin{array}{l}\text { "Yerma", DE F. GARCIA LORCA } \\
\text { (EsPanHA, 1898-1936) } \\
\end{array}$ & ANTONIO ABUJAMrA \\
\hline 1974 & $\begin{array}{l}\text { "O QUE LEVA BOFETADAS", DE LEONID ANDREIEV } \\
\text { (RÚSSIA, 1871/ FINLÂNDIA, 1919) }\end{array}$ & Antonio AbuJAmRA \\
\hline 1974 & $\begin{array}{c}\text { “O CEGO”, DE D. H. LAWRENCE } \\
\text { (INGLATERRA, } 1885 \text { - FranÇA, 1930) }\end{array}$ & FERnando Faro \\
\hline 1974 & $\begin{array}{c}\text { "O ENFERMEIRO", DE MACHADO DE Assis (RJ, } \\
1839 \text { - 1908) }\end{array}$ & $\begin{array}{l}\text { Cassiano Gabus } \\
\text { Mendes }\end{array}$ \\
\hline 1974 & $\begin{array}{c}\text { "O chá das 4", de Cassiano Gabus Mendes } \\
\text { (SP, 1927 - 1993) }\end{array}$ & $\begin{array}{l}\text { Cassiano Gabus } \\
\text { MENDES }\end{array}$ \\
\hline 1974 & $\begin{array}{c}\text { "A CASA DA RUA TURK", DE } \\
\text { DASHIELL HAMMETT (EUA, } 1894-1961)\end{array}$ & FERNANDO FARO \\
\hline 1974 & $\begin{array}{l}\text { "A LIÇÃO", DE EUGENE IONESCO (ROMÊNIA, } \\
1912 \text { - PARIS, 1994) }\end{array}$ & ANTONIO ABUJAMrA \\
\hline 1974 & $\begin{array}{l}\text { "Vestido de Noiva", De Nelson Rodrigues } \\
\text { (RE, } 1912 \text { - RJ, 1980) }\end{array}$ & ANTUNES FiLHO \\
\hline
\end{tabular}




\begin{tabular}{|c|c|c|}
\hline 1974 & $\begin{array}{c}\text { "O LADRÃO", DE Graciliano RAMOS (AL, } \\
1892 \text { - RJ, 1953) }\end{array}$ & $\begin{array}{l}\text { Cassiano Gabus } \\
\text { Mendes } \\
\end{array}$ \\
\hline 1974 & $\begin{array}{l}\text { "O BANQUETE", DE LUCIA BENEDETTI (SP, } \\
1914 \text { - RJ, 1998) }\end{array}$ & Antunes FiLho \\
\hline 1974 & $\begin{array}{c}\text { "O MILAGRE", DE F. WERFFEL (ÁUSTRIA, SEM } \\
\text { INF.) }\end{array}$ & Walter G. Durst \\
\hline 1975 & $\begin{array}{c}\text { "ONDE A CRUZ ESTÁ MARCADA", DE } \\
\text { Eugene O'NeILl (EUA, } 1888-1953 \text { ) }\end{array}$ & AnTONIO AbUjamra \\
\hline 1975 & $\begin{array}{c}\text { "A CASA FECHADA", DE ROBERTO GOMES (SC, } \\
1882 \text { - (SEM INF.)1922) }\end{array}$ & ANTUNES FILHO \\
\hline 1975 & $\begin{array}{c}\text { "Os PEIXES BANANA", DE J.D. SALINGER } \\
\text { (EUA, 1912 - ) }\end{array}$ & FERNANDO FARO \\
\hline 1975 & \begin{tabular}{|c|} 
"A CARTA", DE William SOMERSET MaUGAHM \\
(InglaterRa, $1874-1965$ ) \\
\end{tabular} & Antonio Abujamra \\
\hline 1975 & $\begin{array}{l}\text { "Alô, AlguÉm aI ?", DE William Saroyan } \\
\text { (EUA, } 1905 \text { - 1981) }\end{array}$ & ANTUNES FiLho \\
\hline 1975 & $\begin{array}{l}\text { “Gata EM TETO DE ZINCO QUENTE", DE } \\
\text { Tenesse Williams (EUA, } 1911 \text { - 1983) }\end{array}$ & KIKO JAESS \\
\hline 1975 & $\begin{array}{c}\text { "VenHa Ver O POR- DO- SOL", DE } \\
\text { Lygia Fagundes Telles (SP, } 1923 \text { - ) }\end{array}$ & $\begin{array}{l}\text { Cassiano Gabus } \\
\text { Mendes }\end{array}$ \\
\hline 1975 & $\begin{array}{c}\text { "ACidente EM SumaÚMA", DE J.J. VeIGA } \\
\text { (GO, } 1915 \text { - 1999) }\end{array}$ & FERNANDO FARO \\
\hline 1975 & 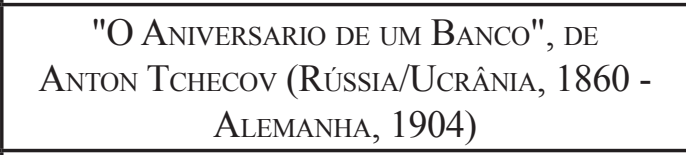 & Antonio AbujamRa \\
\hline 1975 & $\begin{array}{c}\text { "Sem fala, com Fala", DE Walter George } \\
\text { Durst (SP, } 1922 \text { - 1997) }\end{array}$ & $\begin{array}{l}\text { WALTER GEORGE } \\
\text { DuRST } \\
\end{array}$ \\
\hline 1975 & $\begin{array}{c}\text { “A EsPERANÇA", DE PADDY CHAYEFSKY } \\
\text { (EUA, } 1923 \text {-1981) }\end{array}$ & Antonio AbUjamra \\
\hline 1975 & $\begin{array}{c}\text { "O CASO DE DEZ NeGRINHOs", DE } \\
\text { Agatha Christie (InGLATERRa } 1980 \text { - 1976) }\end{array}$ & Ademar Guerra \\
\hline 1975 & $\begin{array}{c}\text { "A CAMA", DE LyGia BoJUNGA Nunes ( RS, } \\
1932 \text { - ) }\end{array}$ & Benjamin Cattan \\
\hline 1975 & $\begin{array}{c}\text { "CORPO FECHADO", DE GUIMARÃEs RoSA } \\
\text { (MG, } 1908 \text { - RJ, 1967) } \\
\end{array}$ & Lima Duarte \\
\hline 1975 & $\begin{array}{l}\text { "TERRA NATAL", DE OdUVALDO ViAnNA (PR, } \\
1892 \text { - RJ, 1972) }\end{array}$ & AdEMAr Guerra \\
\hline 1975 & $\begin{array}{c}\text { "A festa Acabou", de Álvaro Martins (CE, } \\
1868 \text { - 1906) }\end{array}$ & A. C. AssumpÇ̃̃o \\
\hline 1975 & $\begin{array}{c}\text { "A ceia dos CARDEAis", DE JUlio DanTAS } \\
\text { (Portugal, } 1867 \text { - 1962) } \\
\end{array}$ & Nydia Licia \\
\hline 1975 & $\begin{array}{l}\text { "AMORES ANTIGOS", DE MACHADO DE Assis } \\
\text { (RJ, } 1839 \text {-1908) }\end{array}$ & $\begin{array}{l}\text { ANTONIO GHIGO- } \\
\text { NETTO }\end{array}$ \\
\hline 1975 & $\begin{array}{l}\text { "Hugo, O Boneco", De Alberto Cavalcanti } \\
\text { (RJ, } 1897 \text { - FranÇA, 1982) }\end{array}$ & Benjamin Cattan \\
\hline 1975 & $\begin{array}{l}\text { "SOROCO, SUA MÃE, SUA FILHA", DE } \\
\text { Guimarães Rosa (MG, } 1908 \text { - RJ, 1967) }\end{array}$ & KIKO JAESS \\
\hline
\end{tabular}




\begin{tabular}{|c|c|c|}
\hline 1975 & $\begin{array}{l}\text { "GENTE COMO A GENTE", DE RoBERTO FREIRE } \\
\text { (SP, } 1927-2008 \text { ) } \\
\end{array}$ & Ademar Guerra \\
\hline 1975 & $\begin{array}{c}\text { "Felisberto do CAFÉ", DE GASTÃo TOJeIRO } \\
\text { (RJ, 1880/ (SEM INF.)1921) }\end{array}$ & Benedito Corsi \\
\hline 1975 & $\begin{array}{c}\text { "A ESCADA", DE JORGE DE ANDRADE } \\
\text { (SP, } 1922 \text { - 1984) }\end{array}$ & Antunes FiLHo \\
\hline 1975 & $\begin{array}{l}\text { "As ROSAS RUBRAS DA MORTE", DE } \\
\text { JoRge KarAM A PARTIR DE LENDA POPULAR } \\
\text { RECOLHIDA POR SIMÃo LOPES Neto }\end{array}$ & Heloisa Castellar \\
\hline 1975 & $\begin{array}{c}\text { "Do tamanho de um DEFunTO", DE MillôR } \\
\text { FERNANDES (RJ, } 1924 \text { - ) }\end{array}$ & $\begin{array}{c}\text { ANTONIO } \\
\text { C. AsSUMPÇÃo }\end{array}$ \\
\hline 1975 & $\begin{array}{l}\text { "SONATA", DE ÉRICO VERÍSSIMO (RS, 1905- } \\
\text { 1975) }\end{array}$ & KIKO JAESS \\
\hline 1975 & $\begin{array}{l}\text { "UM CASO EXTRAORDINÁRIO", DE EUGENIO } \\
\text { Heltai (HuNGRIA, } 1871 \text { - (SEM INF.), 1957) }\end{array}$ & Antunes FiLho \\
\hline 1975 & $\begin{array}{l}\text { "Vejo a lua no CÉU", de MarQues Rebelo } \\
\text { (RJ, } 1907 \text { - 1973) }\end{array}$ & KIKO JAESS \\
\hline 1975 & "EleCtrA", DE EuRIPEDES (485 - 406 A. C.) & ADEMAR GUERRA \\
\hline 1975 & $\begin{array}{l}\text { "Processo CRISPIM", DE LuIS GUTTEMBERG } \\
\text { (AL,1937 - ) }\end{array}$ & SuZANa Amaral \\
\hline 1975 & $\begin{array}{c}\text { "DA ARTE DE BEM GOVERNAR", AUTO MEDIEVAL } \\
\text { ANÔNIMO }\end{array}$ & AdEMAr GUERra \\
\hline 1975 & $\begin{array}{c}\text { “CAIXA FORTE", DE ARTHUR HAILEY (INGLA- } \\
\text { TERRA, 1920- BAHAMAS, 2004) }\end{array}$ & $\begin{array}{l}\text { Silvio de AbREu E } \\
\text { Eloy de ARAúJo }\end{array}$ \\
\hline 1975 & $\begin{array}{c}\text { "O escravo", De LuCIO CARDOSO (MG, } 1913 \\
\text { - RJ, 1968) }\end{array}$ & KIKO JAESS \\
\hline 1975 & $\begin{array}{l}\text { "SOMOS TODOS DO JARDIM DA INFÂNCIA", DE } \\
\text { DomingOS DE OlIVEIRA (RJ, } 1926 \text { - ) }\end{array}$ & Antunes FiLHo \\
\hline 1976 & $\begin{array}{c}\text { "VERSO E REVERSO", DE JoSÉ DE AlENCAR } \\
\text { (CE, 1829 - RJ, 1877) }\end{array}$ & KIKO JAESS \\
\hline 1976 & $\begin{array}{l}\text { “A viÚVA AZUL”, DE MARCoS ReY (SP, } 1925 \\
-1999)\end{array}$ & $\begin{array}{l}\text { Walter George } \\
\text { Durst }\end{array}$ \\
\hline 1976 & $\begin{array}{l}\text { "Lua Amarela", De Lygia FAgundes Telles } \\
\text { (MA, } 1855 \text { - RJ,1908) }\end{array}$ & Antunes FiLho \\
\hline 1976 & $\begin{array}{c}\text { "Beata Maria do EGito", DE } \\
\text { Raquel de Queiroz (CE, } 1910 \text { - RJ,2003) }\end{array}$ & KIKO JAESS \\
\hline 1976 & $\begin{array}{l}\text { "DEMorado ADEUs", DE TeNESSE Williams } \\
\text { (EUA, } 1911 \text { - 1983) }\end{array}$ & Antunes FiLho \\
\hline 1976 & $\begin{array}{c}\text { "O desembestado", DE ARIOVALdo Mattos } \\
(\mathrm{BA}, 1926-)\end{array}$ & Antunes FiLho \\
\hline 1976 & $\begin{array}{c}\text { "A fronteira", De Menotti del Picchia (SP, } \\
189 \text { 2- 1988) }\end{array}$ & EMílio Fontana \\
\hline 1976 & $\begin{array}{c}\text { "O ciúMES DE UM PEDESTRE", DE } \\
\text { Martins PENA (RJ, } 1815 \text { - LisBOA, 1848) }\end{array}$ & Ademar Guerra \\
\hline 1976 & $\begin{array}{l}\text { "Volte Para CASA, DanNY", DE Marcos Rey } \\
\text { (SP, 1925 - 1999) }\end{array}$ & KIKO JAESS \\
\hline
\end{tabular}




\begin{tabular}{|c|c|c|}
\hline 1976 & $\begin{array}{c}\text { "O DESCONHECIDO", DE OZUALDO CANDEIAS } \\
\text { (SP, } 1918-2007)\end{array}$ & Ozualdo Candeias \\
\hline 1976 & $\begin{array}{l}\text { "O velho Nô", de José Conde (PE, } 1918 \text { - } \\
\text { RJ, 1971) }\end{array}$ & ROBERTO VignATTI \\
\hline 1976 & $\begin{array}{l}\text { "NAPOLEÃO E ElVIRA... OU A TRISTE SORTE DE } \\
\text { UMA PECADORA", LITERATURA DE CORDEL (BR.) }\end{array}$ & AdEMAR GuERra \\
\hline 1976 & $\begin{array}{l}\text { "CORPO A CORPO", DE OdUVALdo Viana FilHo } \\
\text { (RJ, } 1936 \text { - 1974) }\end{array}$ & ANTUNES FiLho \\
\hline 1976 & 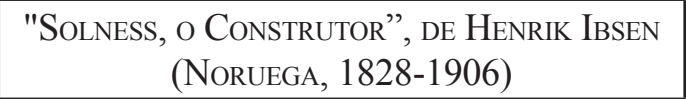 & Antunes FiLHo \\
\hline 1976 & $\begin{array}{c}\text { "O PIANO", DE ANÍBAL MACHADO (MG, } 1894 \\
- \text { RJ, 1964) }\end{array}$ & Roberto Vignatti \\
\hline 1976 & 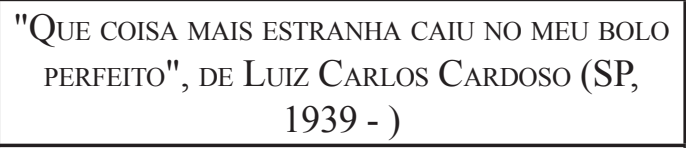 & Antunes FiLho \\
\hline 1976 & $\begin{array}{l}\text { "A Dama de Copas e o Rei de CubA", DE } \\
\text { Timochenco Wehbi (SP, } 1943 \text { - 1986) }\end{array}$ & Silvio de Abreu \\
\hline 1976 & $\begin{array}{c}\text { "Nosferatu ", De JosÉ Vicente (MG, } 1945 \\
-2007 \text { ) } \\
\end{array}$ & AdEMAR GUERRA \\
\hline 1976 & $\begin{array}{l}\text { "Cordiais SAUdaÇões Mr. Kissinger", DE } \\
\text { Lenita Plonczynski (BR., SEM INF.) }\end{array}$ & Antunes FiLHo \\
\hline 1976 & $\begin{array}{c}\text { "ImPlosão", de Consuelo de CAstro (MG, } \\
1946-\text { ) }\end{array}$ & Antunes FiLho \\
\hline 1976 & $\begin{array}{l}\text { "Ultimo capítulo", de Consuelo de Castro } \\
\text { (MG, 1946- ) }\end{array}$ & Roberto Vignatti \\
\hline 1976 & $\begin{array}{l}\text { "O GRAVADOR", DE RUBEM FonSECA. (MG, } \\
1925-)\end{array}$ & RoBerto VignATti \\
\hline 1976 & $\begin{array}{l}\text { "TUDO OU NADA", DE BENEDITO CORSI (BR., } \\
\text { SEM INF.) }\end{array}$ & Antunes Filho \\
\hline 1976 & $\begin{array}{l}\text { "O MISTÉRIO DAS FIGURAS DE BARRO", DE } \\
\text { OSMAN LINS (PE, } 1924 \text { - SP, 1978) } \\
\end{array}$ & KIKO JAESS \\
\hline 1976 & $\begin{array}{c}\text { "HoJE É DIA DE ROCK", DE José VICENTE (MG, } \\
1945 \text { - ) }\end{array}$ & Silvio de ABreu \\
\hline 1976 & $\begin{array}{l}\text { "RELATÓRIO CONFIDENCIAL", DE } \\
\text { DeONísIo da Silva (SC, } 1948 \text { - ) }\end{array}$ & ANTUNES FiLHO \\
\hline 1976 & $\begin{array}{l}\text { "NingUÉM MORRE DUAS VEZES", DE } \\
\text { Luiz LoPES COELHO (SP, 1911-1975) } \\
\end{array}$ & RoBERTO VignATTI \\
\hline 1976 & 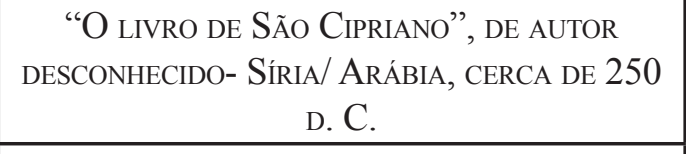 & AdEMAR GUERRA \\
\hline 1976 & “O BILHAR”, DE TÁcITO Rocha (BR., SEM INF.) & EMÍLIO FONTANA \\
\hline 1976 & "Guarania", DE Chico de Assis (SP, 1933 - ) & AdEMAR GUERRA \\
\hline 1976 & $\begin{array}{c}\text { “ChapÉu PRETO, NOIVA BRANCA", DE A. } \\
\text { C.CARVALHO (BR., SEM INF.) }\end{array}$ & AntUNES FiLho \\
\hline 1976 & $\begin{array}{l}\text { "Flores PARA OS MORTOS", DE TENESSE } \\
\text { Williams (EUA, } 1911-1983 \text { ) }\end{array}$ & KIKO JAESS \\
\hline
\end{tabular}




\begin{tabular}{|c|c|c|}
\hline 1976 & $\begin{array}{c}\text { "BABy deVIL", de CAIO Fernando Abreu } \\
\text { (RS, } 1948 \text { - 1996) }\end{array}$ & AdEMAr Guerra \\
\hline 1976 & $\begin{array}{l}\text { “CRIMe e CAstigo", de F. Dostoleviski } \\
\text { (RÚssia } 1821-1881 \text { ) }\end{array}$ & Antunes FiLho \\
\hline 1976 & "O OLHO" DE J. AlVIM (SEM INF.) & ANTUNES FiLHo \\
\hline 1976 & $\begin{array}{c}\text { “Parque Post Meridium", de KiKo Jaess } \\
\text { (Br., SEM INF.) }\end{array}$ & KIKO JAESS \\
\hline 1976 & $\begin{array}{c}\text { "HistóRIA DE EsMERALDINA E OTACIANO", DE } \\
\text { J.S.AMORIM (SEM INF.) }\end{array}$ & AdEMAr GuERra \\
\hline 1976 & $\begin{array}{c}\text { "HoJE SOU UM E AMANHÃ OUTRO", DE QORPO } \\
\text { SANTO (RS, 1829-1883) }\end{array}$ & Antunes FiLho \\
\hline 1977 & $\begin{array}{c}\text { "Noite NA TAVERNA", DE } \\
\text { Álvares de AzEVEDo (SP, } 1831 \text { - RJ, 1852) }\end{array}$ & AdEMAr Guerra \\
\hline 1977 & $\begin{array}{l}\text { "SENHORITA JULIA", DE A. STRINDBERG } \\
\text { (SuÉCIA } 1849-1912 \text { ) }\end{array}$ & Antunes FiLHo \\
\hline 1977 & $\begin{array}{l}\text { "O ANJo RAFAEL", DE MACHADO DE Assis } \\
\text { (RJ, 1839-1908) }\end{array}$ & KIKO JAESS \\
\hline 1977 & $\begin{array}{l}\text { "CAso clínico", de José de Auigusto (SEM } \\
\text { INF.) }\end{array}$ & Antunes FiLHo \\
\hline 1977 & $\begin{array}{c}\text { "Viva Olegário", de Luis CARLos Cardoso } \\
\text { (SP, } 1939 \text { - ) }\end{array}$ & Antunes FiLho \\
\hline 1977 & $\begin{array}{c}\text { “ReveilloN", de Flavio Márcio (MG, } 1945 \\
\text { - SP, 1979) }\end{array}$ & Antunes FiLho \\
\hline 1977 & $\begin{array}{c}\text { “Terror e miséria no III Reich", De B. Bre- } \\
\text { Cht (Alemanha, } 1898-1956)\end{array}$ & AdEMAr GuERra \\
\hline 1977 & $\begin{array}{l}\text { "O AQUÁRIo", DE OsWALDo BARRETO (SEM } \\
\text { INF.) }\end{array}$ & ROBERTO VignATTI \\
\hline 1977 & $\begin{array}{l}\text { "Raízes", de Domingos Pellegrini Junior } \\
(\mathrm{PR}, 1949 \text { - ) }\end{array}$ & AdEMAr Guerra \\
\hline 1977 & "DivisA", DE ChICO DE Assis (SP, 1933 - ) & AdEMAR GUERRA \\
\hline 1977 & $\begin{array}{l}\text { "O visitante”, DE Hilda HiLst (SP,1930- } \\
\text { 2004) }\end{array}$ & KIKO JAESS \\
\hline 1977 & $\begin{array}{c}\text { "DiA toRto", DE ENIO GonÇALVES (BR., SEM } \\
\text { INF.) }\end{array}$ & Antonio ABujamra \\
\hline 1977 & “A RAiz do Grito”, A. Cláudio (SEM INF.) & $\begin{array}{l}\text { Walter George } \\
\text { Durst }\end{array}$ \\
\hline 1977 & $\begin{array}{l}\text { "UM PÁSSARO NO MEU OMBRO”, DE } \\
\text { A.C. CARVALHO (BR., SEM INF.) }\end{array}$ & $\begin{array}{l}\text { WaLTER GEORGE } \\
\text { DuRST }\end{array}$ \\
\hline 1977 & $\begin{array}{c}\text { “AMORES E LICORES”, DE ElísIo AlbuQUER- } \\
\text { QUE ( BR, SEM INF., 1920-1983) }\end{array}$ & Antonio ABujamra \\
\hline 1977 & “HAPPY END”, dE A. DE PÁdUA (BR., SEM INF.) & $\begin{array}{l}\text { FERNANDO PACHECO } \\
\text { JoRDÃo }\end{array}$ \\
\hline 1978 & $\begin{array}{l}\text { "NAU CATRINETA", DE RUBEM FonsECA (MG, } \\
1925-)\end{array}$ & ANTUNES FiLHO \\
\hline 1978 & $\begin{array}{l}\text { "StrindBerg - Duas estórias: A mais FORTE } \\
\text { E Paria", de A. StrindBerg (SuÉCIA, } 1849 \text { - } \\
\text { 1912) }\end{array}$ & Antunes FiLho \\
\hline
\end{tabular}




\begin{tabular}{|c|c|c|}
\hline 1978 & $\begin{array}{c}\text { “Quebranto", De Coelho Neto (MA,1864 } \\
\text { - RJ,1934) }\end{array}$ & AdEMAr Guerra \\
\hline 1978 & $\begin{array}{c}\text { "RANCOR", DE JOHN OsbORNE (INGLATERRA, } \\
1929,1994)\end{array}$ & AdEMAR GuERra \\
\hline 1978 & $\begin{array}{c}\text { "Retrato de UMA SOLTEIRONA", DE TENESSE } \\
\text { Williams (EUA, } 1911-1983 \text { ) }\end{array}$ & ANTUNES FiLHo \\
\hline 1978 & $\begin{array}{l}\text { “AdÃo e Eva e outros membros da famí- } \\
\text { LiA”, De Állvaro Moreyra (RS, } 1888 \text { - } \\
\text { RJ.,1964) }\end{array}$ & AdEMAR GUERRA \\
\hline 1978 & $\begin{array}{c}\text { “A ILHA DAS CABRAS", DE UGO BETTI (ITÁLIA, } \\
1892-1953)\end{array}$ & RoBerto VignatTI \\
\hline 1978 & $\begin{array}{c}\text { “O TORNIQUETE”, DE LUIGI PIRANDELLO (ITÁ- } \\
\text { LIA, } 1807 \text { - 1936) }\end{array}$ & ANTUNES FiLHo \\
\hline 1978 & $\begin{array}{c}\text { "O BeSOURO NEGRO", DE BRUNO FranK (Ale- } \\
\text { MANHA, } 1878 \text { - USA, 1945) }\end{array}$ & Antonio Abujamra \\
\hline 1978 & $\begin{array}{c}\text { “The StAR", De JuAn CARLos Gene (ARGen- } \\
\text { TINA, SEM INF) }\end{array}$ & Antonio AbujamRa \\
\hline 1979 & $\begin{array}{l}\text { "Os ANJos", DE BERTHOLD BRECHT (AlEMA- } \\
\text { NHA, } 1898-1956)\end{array}$ & I. MoRELLI \\
\hline 1979 & "Nó CEGO", DE C. VEREZA (SEM INF.) & (SEM INF.) \\
\hline 1979 & $\begin{array}{c}\text { “A MÃE E O FILHO DA MÃE”, DE WANDER PIRO- } \\
\text { LI (MG, 1933-) }\end{array}$ & RoBerto VignATti \\
\hline 1979 & $\begin{array}{c}\text { “TudO EM TUDO”, COMPILAÇão DE VÁRIOS } \\
\text { AUTORES ESTRANGEIROS POR M. VINÍCIUS E O. } \\
\text { PETTI }\end{array}$ & ÍtALO MORELli \\
\hline 1985 & $\begin{array}{l}\text { “UM HAMLET”, DE G. TeStORI (ItÁlia, } 1923 \\
-1993 \text { ) }\end{array}$ & Antonio AbUjamra \\
\hline 1985 & $\begin{array}{c}\text { "Bye, BYe POROROCA", De MAH Lully (BR., } \\
\text { SEM INF.) E TimochenKo WeHBI (SP, } 1943 \text { - } \\
1986 \text { ) }\end{array}$ & AdEMAr Guerra \\
\hline 1985 & $\begin{array}{c}\text { "SENHORA DOS AFOGADOS", DE NELSON Ro- } \\
\text { DRIGUES (RE, } 1912 \text { - RJ, 1980) }\end{array}$ & ANTONIO ABUJAMra \\
\hline
\end{tabular}

\section{Observações finais}

A listagens dos 122 títulos que cons-tituíram o total das exibições do programa Teatro 2 nos conduzem a cinco observações gerais:

1) Embora tenha havido a participação de vários diretores no conjunto dos teleteatros do Teatro 2 , os diretores com maior número e com maior regularidade de participações foram quatro: Antunes Filho (com
29 direções), Ademar Guerra (20), Antonio Abujamra (15) e Kiko Jaess (13). É a esse núcleo de diretores que se deve atribuir uma certa identidade caracterizadora do programa.

2) Os anos de maior atividade, de maior número de produções exibidas, no Teatro 2 foram os três primeiros anos do programa: 1974, 1975 e 1976. Uma possível explicação para tal fato pode ser que o pro- 
cesso de distensão política, iniciado em 1975, aos poucos foi tornando os palcos teatrais um lugar para o qual teatrólogos, diretores e atores pudessem retornar em segurança e inclusive levar suas experiências do ambiente televisivo. Destacase, quanto a esse último aspecto, a antológica montagem teatral de Antunes Filho, de Macunaíma, de Mário de Andrade, em 1978.

3) Dos 122 teleteatros exibidos pelo Teatro 2 apenas 34 foram de autoria de escritores estrangeiros. Destes, a grande maioria é composta por contemporâneos, como, Tenesse Willians, Eugene O'Neill Pirandello, Ionesco e Brecht, atestando o quanto esse grupo de diretores estava aberto para o que de melhor qualidade e de maior grau de questionamento e envolvimento internacional havia no panorama teatral mundial.

4) Nos 88 teleteatros, baseados em textos de autores nacionais, nota-se uma tendência maior para o contemporâneo e uma abertura para vários segmentos culturais e de propostas estéticas passando pelos clássicos Machado de Assis e Guimarães Rosa (aí incluindo também o hoje clássico Nelson Rodrigues), pelo experimentalismo de J.J. Veiga, pela literatura mais próxima da chamada paraliteratura de Millôr Fernandes e Luiz Lopes Coelho e pela literatura popular oral, os chamados cordéis.

5) Embora a grande maioria dos teleteatros tenham tido sua origem em textos teatrais, outros gêneros e formatos literários também foram utilizadas como fonte para o Teatro 2. Nesse sentido notamos, entre outros, a presença da adaptação de contos, por exemplo, "Soroco, sua mãe, sua filha", de Guimarães Rosa, adaptado e dirigido por Kiko Jaess; de lendas, como no teleteatro "As rosas rubras da morte", de Jorge Karam, a partir de lenda popular recolhida por Simão Lopes Neto; de cordel, no teleteatro "Napoleão e Elvira... Ou a triste sorte de uma pecadora", dirigido por Ademar Guerra, o que demonstra também a abertura estética do programa.

\section{Referências}

BRANDÃO FARIA, Cristina. O Grande teatro Tupi do Rio de Janeiro. Juiz de Fora: UFJF, 2005.

BRANDÃO FARIA, Maria Cristina. Paper: O Grande teatro Tupi do Rio de Janeiro. In: CD INTERCOM - Sociedade Brasileira de Estudos Interdisciplinares da Comunicação XXV Congresso Brasileiro de Ciências da Comunicação - Salvador/BA - 1 a 5 Set 2002.

COSTA, Alcir H. da; SIMÕES, Inimá Ferreira e KEHL, Maria Rita. Um País no ar: História da TV brasileira em 3 canais. São Paulo: Brasiliense/Funarte, 1986

FERRARA, Lucrecia (coord). Da Literatura à tevê. São Paulo: Se- 
cretaria Municipal de Cultura, departamento de informação e Documentação Artísticas, Centro de Documentação e Informação sobre arte Brasileira Contemporânea, 1981

KEHL, Maria Rita. Eu vi um Brasil na TV. In: COSTA, Alcir H. da; SIMÕES, Inimá Ferreira e KEHL, Maria Rita. Um País no ar: História da TV brasileira em 3 canais. São Paulo: Brasiliense/Funarte, 1986, págs. 167-276.

MANOEL CARLOS. O Grande teatro. Livreto de apresentação da exposição O Grande Teatro Tupi. Caixa Econômica Fedeal. Exposição de 31 de maio de 2005 a 31 de julho de 2005. Curadoria: Anna Vacchiano.

ORTIZ, Renato, BORELLI, Silvia e RAMOS, J.M. Telenovela: história e produção. São Paulo, Brasiliense, 1991, 2a . edição

PAllotTINI, Renata. Dramaturgia da televisão. São Paulo: Ed. Moderna, 1998.

PRIOLLI, Gabriel BORELLI, Silvia. A Deusa Ferida: Por que a Rede Globo não é mais a campeã absoluta de audiência. São Paulo: Summus ed., 2000.

PORTO e SILVA, Flavio Luiz (coord). O teleteatro paulista nas décadas de 50 e 60. São Paulo: Secretaria Municipal de Cultura, 1981.

RAMOS, J. M. O e BORELLI, Silvia. A telenovela diária. In: Ortiz, Renato, Borelli, Silvia e Ramos, J.M. Telenovela: história e produção. São Paulo: Brasiliense, 1991, 2a . edição,

REIMÃO, Sandra. Em Instantes. Notas sobre a programação na TV brasileira (1965-1995). São Paulo: Editora Cabral, 1997. Não aparece no texto.

SIMÕES, Inimá. "TV à Chateaubriand" In. COSTA, Alcir H. da; SIMÕES, Inimá Ferreira e KEHL, Maria Rita. Um País no ar: História da TV brasileira em 3 canais. São Paulo: Brasiliense/Funarte, 1986

XAVIER, Ricardo (Rixa). Almanaque da TV: 50 anos de memória e informação. Rio de Janeiro: Editora Objetiva, 2000.

TV CULTURA. Portal <www.tvcultura.com.br>. Acesso em junho 2007 e agosto 2008. 